\title{
Article
}

\section{MOFs for the Sensitive Detection of Ammonia: Deployment of fcu-MOF Thin-Films as Effective Chemical Capacitive Sensors.}

\author{
Ayalew Hussen Assen, Omar YASSINE, Osama Shekhah, Mohamed Eddaoudi, and Khaled N. Salama
}

ACS Sens., Just Accepted Manuscript • DOI: 10.1021/acssensors.7b00304 • Publication Date (Web): 15 Aug 2017

Downloaded from http://pubs.acs.org on August 20, 2017

\section{Just Accepted}

"Just Accepted" manuscripts have been peer-reviewed and accepted for publication. They are posted online prior to technical editing, formatting for publication and author proofing. The American Chemical Society provides "Just Accepted" as a free service to the research community to expedite the dissemination of scientific material as soon as possible after acceptance. "Just Accepted" manuscripts appear in full in PDF format accompanied by an HTML abstract. "Just Accepted" manuscripts have been fully peer reviewed, but should not be considered the official version of record. They are accessible to all readers and citable by the Digital Object Identifier (DOI®). "Just Accepted" is an optional service offered to authors. Therefore, the "Just Accepted" Web site may not include all articles that will be published in the journal. After a manuscript is technically edited and formatted, it will be removed from the "Just Accepted" Web site and published as an ASAP article. Note that technical editing may introduce minor changes to the manuscript text and/or graphics which could affect content, and all legal disclaimers and ethical guidelines that apply to the journal pertain. ACS cannot be held responsible for errors or consequences arising from the use of information contained in these "Just Accepted" manuscripts. 


\begin{abstract}
This work reports on the fabrication and deployment of a select metal-organic framework (MOF) thin film as an advanced chemical capacitive sensor for the sensing/detection of ammonia $\left(\mathrm{NH}_{3}\right)$ at room temperature. Namely, the MOF thin film sensing layer consists of a rare-earth (RE) MOF (RE-fcu-MOF) deposited on a capacitive interdigitated electrode (IDE). Purposely, the chemically stable naphthalene-based RE-fcu-MOF (NDCY-fcu-MOF) was elected and prepared/arranged as a thin film on a pre-functionalized capacitive IDE via the solvothermal growth method. Unlike earlier realizations, the fabricated MOF-based sensor showed a notable detection sensitivity for $\mathrm{NH}_{3}$ at concentrations down to $1 \mathrm{ppm}$, with a detection limit appraised to be around $100 \mathrm{ppb}$ (at room temperature) even in the presence of humidity and/or $\mathrm{CO}_{2}$. Distinctly, the NDC-Y-fcu-MOF based sensor exhibited the required stability to $\mathrm{NH}_{3}$, in contract to other reported $\mathrm{MOFs}$, and a remarkable detection selectivity towards $\mathrm{NH}_{3}$ vs. $\mathrm{CH}_{4}, \mathrm{NO}_{2}, \mathrm{H}_{2}$ and $\mathrm{C}_{7} \mathrm{H}_{8}$. The NDC-Y-fcu-MOF based sensor exhibited excellent performance for sensing ammonia for simulated breathing system in the presence of the mixture of carbon dioxide and/or humidity (water vapor), with no major alteration in the detection signal.
\end{abstract}

Keywords: MOFs, Sensing, Ammonia, Capacitive sensors, thin films.

Our ecosystem comprises various gases/vapors in a relatively definite concentrations, generated as result of unceasing volcanic eruptions that overcame our planet, including greenhouse gases (e.g. water vapor, methane, carbon dioxide) and various other harmful gases such as carbon monoxide and ammonia. ${ }^{1}$ Nonetheless, today the concentration of ammonia $\left(\mathrm{NH}_{3}\right)$ in the atmosphere is increasing as a result of direct or indirect human activities. Manifestly, the global emission of $\mathrm{NH}_{3}$ has almost doubled in the last 40 years, from 25 million tons in the 1970's to approximately 50 million tons in recent years. ${ }^{2-5}$ Perceptibly, the non-controlled release/increase of $\mathrm{NH}_{3}$ in our atmosphere represents a serious threat to the suitability of our ecosystem, e.g. promoting unwarranted particulate formation, climate change with multiple associate health side effects. ${ }^{3}$

Recognizably, three major sources contribute to the release of $\mathrm{NH}_{3}$ in the atmosphere. The first is atmospheric deposition, which mainly entails the direct deposition of ammonium and nitrate salts via their addition to the soil in the form of dissolved dust or particulates in rain water. ${ }^{6}$ The second source is nitrification by bacterial nitrogen fixation, which is the result of certain bacteria activity through which they bind nitrogen and then release the excess ammonia into the atmosphere. ${ }^{7}$ The third source is associated to the combustion of fossil fuels in both chemical industries and transport sectors. ${ }^{1}$

The ever increasing production of ammonia necessitates: i) in the long term, the development of sustainable technologies limiting the over production/release ammonia, ii) immediate deployment of the necessary means to remediate excess ammonia and critically derive tools to prevent over-exposure to ammonia and in a timely-manner prevent its potential harm by early sensing. Decisively, the effective detection/sensing of $\mathrm{NH}_{3}$ is essential in a variety of technologies/areas, including automotive and environmental sectors, chemical industries, and medical diagnostics. ${ }^{1,8}$ It is to be noted that the human nose fails to detect the unforeseen exposure to low-concentration of ammonia, but unbearably senses effectively the presence of ammonia in a relatively high concentrations due to its very penetrating associated smell. Certainly, the detection of ammonia at low concentrations is critical and in some instances with very high sensitivity/quantified at ultra-low concentrations below parts per billion (ppb) in the air, as required in many fields of applications like for example, in automotive industry (measure $\mathrm{NH}_{3}$ emission from vehicles, passenger cabinet air control and detection of $\mathrm{NH}_{3}$ slip) and in chemical industries as leakage alarm. 8,9

Nowadays, various approaches were deployed to detect/sense different levels of ammonia in various contexts, as described in the open literature. ${ }^{1,8}$ Namely, a variety of sensors are used in the automobile exhaust pipes for measuring the resultant ammonia, at ultra-low concentration levels, and monitoring its subsequent release to the ambient environment. It is to be noted that the most frequently used techniques in commercial ammonia detectors are metal-oxide gas sensors, ${ }^{10-14}$ catalytic ammonia detectors, ${ }^{15,16}$ conducting polymer ammonia analyzers and optical ammonia detection methods. ${ }^{17-20}$ For example, Nayak et al. reported a detection of $400 \mathrm{ppm}$ of ammonia at $300^{\circ} \mathrm{C}$ using mixed metal oxide $\mathrm{WO}_{3}-\mathrm{SnO}_{2}$ hierarchical nanostructured materials. ${ }^{21}$ Polyaniline nanoparticles were used to detect ammonia down to $1 \mathrm{ppm}$ at $80^{\circ} \mathrm{C}^{22}$ Aubrecht et al. developed an optical method for the detection of ammonia, based on the absorption of evanescent waves by 
organometallic reagents, permitting detection at lower ammonia levels of approximately $30 \mathrm{ppm} .{ }^{23}$ Yavari et al. reported a detection of ammonia at $20 \mathrm{ppm}$ by employing continuous nano-sheets of graphene foam. ${ }^{24}$ Attractively, Duy et al. reported a $5 \mathrm{ppm}$ ammonia detection at room temperature using reduced graphene oxide (RGO) films. ${ }^{25}$

Nonetheless, these different types of sensors, which have shown good performance, suffer from some drawbacks associated to their composition (e.g., the metal-oxide sensors lack the desired selectivity to a specific gas) and to their concomitant high operation temperature (e.g., in relation to $\mathrm{WO}_{3}$, which has the lowest ammonia detection down to $1 \mathrm{ppm}$ at an elevated temperature of over $\left.400^{\circ} \mathrm{C}\right){ }^{1}$ In the case of catalytic detectors, the lower detection limit is normally in the low-ppm range and their accuracy is restricted. ${ }^{15}$ For conducting polymer sensors, the irreversible reaction with $\mathrm{NH}_{3}$ results in an increase in the mass of the polymer film, causing the sensitivity of the sensor to decay with continuous exposure to $\mathrm{NH}_{3} \cdot{ }^{17}$

Sensors based on interdigitated electrodes (IDEs) have been gaining more attention in recent years, due to the interesting advantages that they offer over other types of sensors. ${ }^{26-29}$ Capacitive chemical sensors constructed using IDEs offer the prospective to increase the IDE-contact area between the sensing material and the sensor circuitry. ${ }^{26}$ In this case, the chemical sensing layer acts as the dielectric between two parallel IDE. As a result, a capacitive sensors reaction to stimuli is measured by a change in the sensors capacitance. In addition, the ability to miniaturize and lower the cost of IDE sensors offers potential to operate with a low volume of samples and promotes their plausible integration with electronics reasonably simpler. Particularly, IDEs offer a prospect for accessing the requisite low-power sensing platforms, such as lab-on- open chip applications. ${ }^{27-32}$

Metal-organic frameworks (MOFs), organic-inorganic hybrid crystalline porous materials, are composed of single metal ions or metal clusters linked by polytopic organic ligands. MOFs offer unique structural diversity in contrast to other porous materials, allowing the successful control of framework topology, porosity and functionality. ${ }^{33-35}$ MOFs have shown great potential for various applications pertaining to energy and environmental sustainability. ${ }^{33-36}$ Recently, MOFs have been applied as sensitive layers for various gas detection systems. ${ }^{38-40}$ It is to state that the use of a given MOF with adequate pore size/shape is not the sole perquisite for efficient detection of hazardous gases/vapors. Additionally MOF should encompass suitable adsorption sites promoting specific interactions between the harmful adsorbates and the MOF host and subsequently present the desired sensing performence. ${ }^{37}$

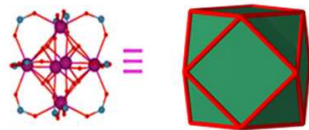

hexanuclear cluster
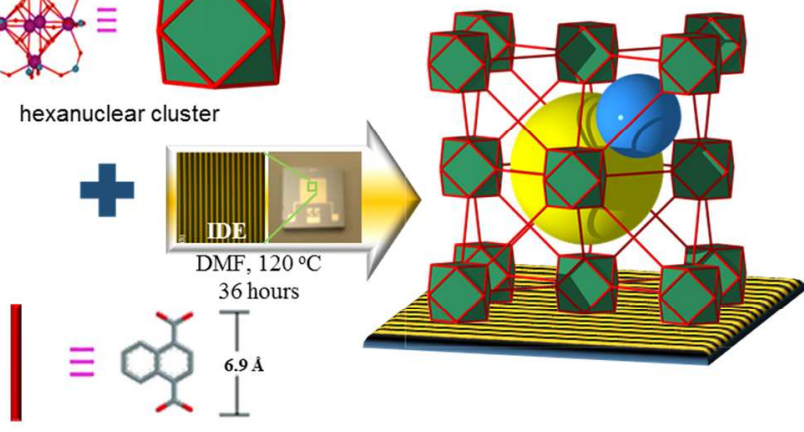

organic linker

Scheme 1. Schematic representation of the optimized solovothermal preparation approach of naphthalene-based fcu-MOF (NDC-Y- fcu-MOF) thin film on IDE substrate.
For example, the presence of metal sites (i.e. coordinative unsaturated metal centers) or certain functionalization on the pore surface may enhance the sensing performance of MOFs for certain toxic compounds via coordination bonds, acidbase/electrostatic interactions. An efficient strategy for the synthesis of materials that preferentially sense a particular gas/vapor is thus to target a particular surface chemistry instead of optimizing the porosity alone.

Successful deployment of MOFs as reliable sensors for $\mathrm{NH}_{3}$ has been hindered/delayed due to the scarcity of stable MOFs upon exposure to ammonia. ${ }^{33}$ Distinctively, Dinca et al. recently reported two types of MOFs with sensing capabilities to $\mathrm{NH}_{3}$ at $100^{\circ} \mathrm{C}$ based on changes in their associated luminescence, but with no noticeable $\mathrm{NH}_{3}$ sensing selectivity at room temperature. ${ }^{41-42}$ Prominently, a rare earth (RE)-based MOF platform with fcu topology (RE-fcu-MOF), an isostructural MOF platform to the zirconium-based UiO-66, has been recently disclosed by Eddaoudi et al. ${ }^{35-37}$ The fcu-MOF platform was constructed using the molecular building block (MBB) approach, ${ }^{36}$ where the 12 coordinated RE hexanuclear MBBs were connected by ditopic ligands, either hetero- or homofunctional and fluorinated or nonfluorinated linkers, in the presence a modulator/structural directing agent (SDA). Importantly, The application of reticular chemistry enabled the fine tuning of the triangular window, the sole entrance to the pore system, aperture size and functionality, by employing shorter and/or bulkier bridging organic ligands, and the subsequent attainment isoreticular RE-fcu-MOFs with fine-tuned adsorption/kinetic properties. ${ }^{35-37}$ In light of the unique adsorption properties of these RE-fcu-MOFs associated with a remarkable thermal and chemical stability, ${ }^{35-37}$ we deposited/grown them as thin films on capacitive IDE substrates and proven their usage as ultra-sensitive sensors for $\mathrm{H}_{2} \mathrm{~S}^{38}$ Rationally, we aimed to extend the prospective sensing capabilities of this unique RE-fcu-MOF platform to include other critical toxic gases such as $\mathrm{NH}_{3} \cdot{ }^{35-38}$ Markedly, the naphthalene (NDC) based RE-fcu-MOF (NDC-Yfcu-MOF) showed exceptional sensing ability for ammonia.

\section{EXPERIMENTAL SECTION}

X-ray diffraction (XRD) measurements, were carried out at room temperature on a PANalyticalX'Pert PRO diffractometer $45 \mathrm{kV}, 40 \mathrm{~mA}$ for $\mathrm{CuK} \alpha(\lambda=1.5418 \AA)$, with a scan rate of $1.0^{\circ}$ $\min ^{-1}$ and a step size of $0.01^{\circ}$ in $2 \theta$. Scanning electron microscope (SEM) characterization was performed using an FEI Quanta 600 field emission SEM (accelerating voltage: $30 \mathrm{kV}$ )

Interdigitated electrodes (IDEs) were fabricated on a silicon wafer. Specifically, a $2 \mu \mathrm{m}$ oxide layer was thermally grown for electrical isolation. A layer of $10 \mathrm{~nm} \mathrm{Ti}$ and $300 \mathrm{~nm}$ Au was first deposited via physical vapor deposition (PVD) in an ESC reactive and metal sputter system. Photolithography was then utilized to pattern the electrodes. The metal layer was patterned by dry etching using the Oxford Instruments PlasmaLab System and the exposed oxide thickness was further verified using a Nanospec 6100 Reflectometer to ensure that the metal layer was properly etched. The IDEs were designed with $4 \mu \mathrm{m}$ fingers and $5 \mu \mathrm{m}$ spaces. Two $\mathrm{Au}$ wires and contact pads were patterned to perform the electrical measurements.

Thin films of the NDC-Y-fcu-MOFs were prepared solvothermally by heating a solution that containing 1, 4-naphthalene dicarboxylic acid acid $(18.8 \mathrm{mg}, 0.087 \mathrm{mmol}), \mathrm{Y}\left(\mathrm{NO}_{3}\right)_{3} \cdot 6 \mathrm{H}_{2} \mathrm{O}(33.4$ $\mathrm{mg}, \quad 0.087 \mathrm{mmol}), 2$-fluorobenzoic acid $(97.5 \mathrm{mg}, 0.70 \mathrm{mmol})$ DMF $(3.0 \mathrm{~mL})$, deionized $\mathrm{H}_{2} \mathrm{O}(0.5 \mathrm{~mL})$ and Nitric acid $(0.4 \mathrm{ml}$ of $4 \mathrm{M}$ solution in DMF), all combined in a $20 \mathrm{~mL}$ scintillation vial. A pre-functionalized IDE chip with the 11-mercaptoundecanol (MUD) self-assembled monolayer (SAM) was placed inside the vial, which was sealed and heated to $115^{\circ} \mathrm{C}$ for $48 \mathrm{~h}$ and then 
cooled to room temperature. The IDE chip was collected and washed with approximately $10 \mathrm{~mL}$ of anhydrous DMF and immersed in $10 \mathrm{~mL}$ of ethanol for three days, during which the ethanol solution was refreshed three times daily.

Gas-sensing tests were performed using a fully automated measurement system with the LabVIEW..$^{39,40}$ The coated sensor was placed inside the detection chamber and connected to the LCR meter to detect the capacitive change. The samples were first activated under vacuum for one hour; then the chamber was purged with pure nitrogen. Nitrogen gas was used as a carrier gas to dilute the $\mathrm{NH}_{3}$ to desired concentrations; ranging from 1 to 100 ppm.

\section{RESULTS AND DISCUSSION}

A series of three isoreticular Y-fcu-MOFs, based on three ditopic ligands namely 2-fluoro-4-(1H-tetrazol-5-yl)benzoic acid $\left(\mathrm{H}_{2}\right.$ FTZB), 1,4-naphth-alenedicarboxylate (1,4-NDC) and fumarate (fum) ${ }^{35-37}$ were tested by exposing the resultant powder material to $\mathrm{NH}_{3}$ vapor at room temperature for various extent of time. The material stability was evaluated/checked using XRD and gas adsorption measurements after exposure to ammonia. The NDC-Y-fcu-MOF was observed to express a prominent stability as confirmed by the XRD studies, where no change in the pattern or decrease in the intensity of the peaks was observed (Figure 1a). Additionally, the $\mathrm{N}_{2}$ adsorption isotherms confirmed the exceptional stability, as no noticeable changes were observed in the shape of the isotherms nor on the uptake amounts after $\mathrm{NH}_{3}$ vapor exposure (Figures 1b).

Certainly, the IDE sensors were fabricated using clean room technologies, following the protocol reported previously. ${ }^{39}$ Prior to the growth of the MOF thin film, the IDEs were firstly functionalized with an OH-terminated MUD SAM. ${ }^{44-50}$ By applying the optimized reaction conditions from the solvothermal growth method for the fabrication of the NDC-Y-fcu-MOF, we were successful in growing a homogenous NDC-Y-fcu-MOF thin film on the IDE support. The fabricated thin film crystallinity was confirmed using X-ray diffraction (XRD) measurements, as shown in Figure 2, which revealed a preferential orientation growth along the [111] direction. ${ }^{32,44-48}$ The thin film morphology was characterized using SEM (see Figure 3), corroborating the formation of a thin film composed of a densely packed NDC-Yfcu-MOF crystals. This approach resulted in the fabrication of the NDC-Y-fcu-MOF thin film with the desired preferential growth orientation, affording the readily exposure of the triangular windows of the NDC-Y-fcu-MOF for the prospective adsorption/inclusion of various guest molecules, such as ammonia the subject of this study. ${ }^{31,34}$

The successful growth of the NDC-Y-fcu-MOF crystals on the IDE substrate affords the ability to monitor and measure the changes in sensing film permittivity upon gas/vapor adsorption. ${ }^{38,39}$ Appropriately, we investigated the sensing properties of NDC-Y-fcu-MOF thin film on capacitive IDEs for $\mathrm{NH}_{3}$ and various other gases/vapors, including methane $\left(\mathrm{CH}_{4}\right)$, propane $\left(\mathrm{C}_{3} \mathrm{H}_{8}\right)$, nitrogen dioxide $\left(\mathrm{NO}_{2}\right)$, hydrogen $\left(\mathrm{H}_{2}\right)$ and toluene $\left(\mathrm{C}_{7} \mathrm{H}_{8}\right)$. The gas sensing tests were performed using a fully automated measurement system with the LabVIEW. ${ }^{39}$ The coated sensor was placed inside the detection chamber and connected to the LCR meter (E4980A) to detect the capacitive change. The samples were first activated under vacuum for one hour; the chamber was later purged with pure nitrogen. Nitrogen gas was used as a carrier gas to dilute the $\mathrm{NH}_{3}$ to the desired concentration.
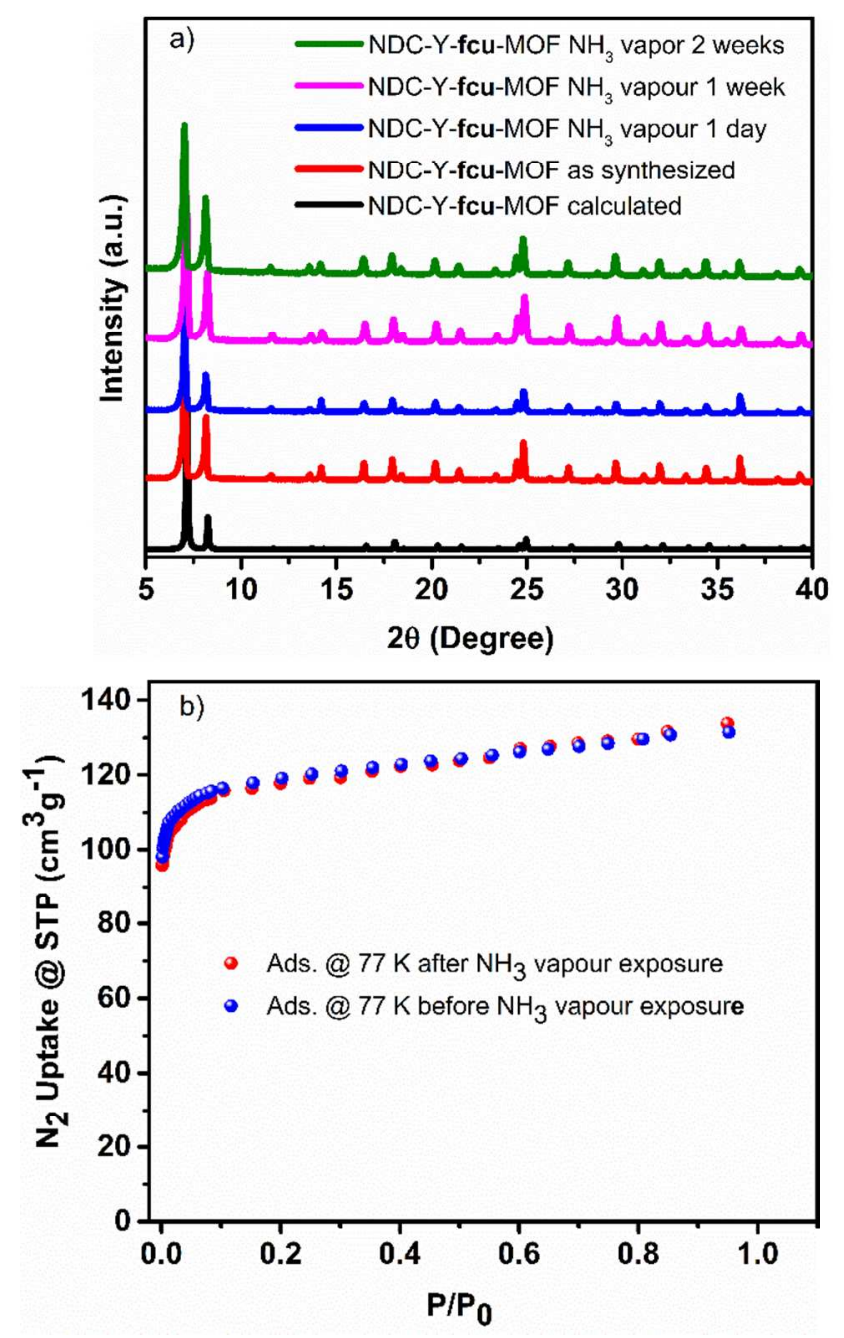

Figure 1. (a) X-ray diffraction (XRD) patterns of NDC-Y-fcuMOF before (black) and after different times of exposure to $\mathrm{NH}_{3}$ vapor. (b) $\mathrm{N}_{2}$ sorption isotherms for NDC-Y-fcu-MOF before (blue) and after (red) exposure to $\mathrm{NH}_{3}$ vapor for 14 days. Encouraged by the evident chemical stability of the NDC-Y-fcu-MOF and its presumed maintained structure integrity in the presence of $\mathrm{NH}_{3}$, we aspired the fabrication/deposition of the NDC-Y-fcuMOF as a thin film to act as the sensing layer on capacitive IDEs (Scheme 1). ${ }^{35-39}$

As depicted in Figure 4, the NDC-Y-fcu-MOF sensor was able to detect $\mathrm{NH}_{3}$ at range from 1 to $100 \mathrm{ppm}$ with a linear response (see Figure 4 inset), with a detection limit of $92 \mathrm{ppb}$, which was calculated based on the root-mean-square deviation method. ${ }^{51}$ The response time of our sensor, which is limited by the gas chamber size, is estimated to be around 250 seconds and be regarded good when compared to other sensors. ${ }^{24,43}$

The stability of the NDC-Y-fcu-MOF capacitive sensor for $\mathrm{NH}_{3}$ detection at room temperature was demonstrated using reproducibility tests, through which the performance of the sensor for detecting two concentrations of $\mathrm{NH}_{3}$, namely 10 and $25 \mathrm{ppm}$, over a testing period of more than two weeks (Figure 5). Clearly, the results showed that the detections levels were steady/stable and uniform, with a negligible variation over the range of various cycles for the tested period of time, attesting to the stability and durability of the NDC-Y-fcu-MOF capacitive sensor for $\mathrm{NH}_{3}$ over this range of tested concentrations. 


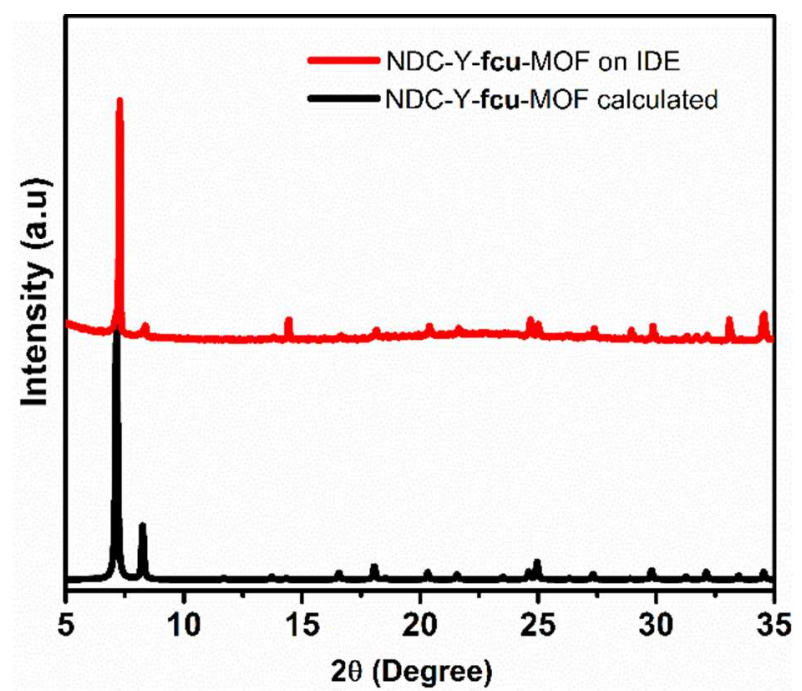

Figure 2. X-ray diffraction (XRD) patterns of NDC-Y-fcu-MOF, (black) calculated and (red) thin film grown on the IDE substrate.

To determine the effect of the relative humidity ( $\mathrm{RH}$ ) over the performance of our sensor, the response behavior of the NDC-Yfcu-MOF thin film sensor was recorded upon exposure to 10 and $25 \mathrm{ppm}$ of $\mathrm{NH}_{3}$, respectively under different $\mathrm{RH}$ levels and at room temperature (Figure 6). The results corroborate the performance of the NDC-Y-fcu-MOF sensor response within different RH levels ranging from 5-85\%, affirming the response of the sensor is stable and unchanged up to $30 \% \mathrm{RH}$ as a result of the strong affinity of the NDC-Y-fcu-MOF sensor to $\mathrm{NH}_{3}$ and the plausible displacement of the water molecules by $\mathrm{NH}_{3}$. However with increasing the RH levels above $30 \%$, we observe a relatively small increase with the sensor response, which may be attributed to the dissolution of more $\mathrm{NH}_{3}$ molecules into physisorbed water on the MOF sensing layer. A similar behavior is observed in other ammonia and gas sensors. ${ }^{25,52,53}$

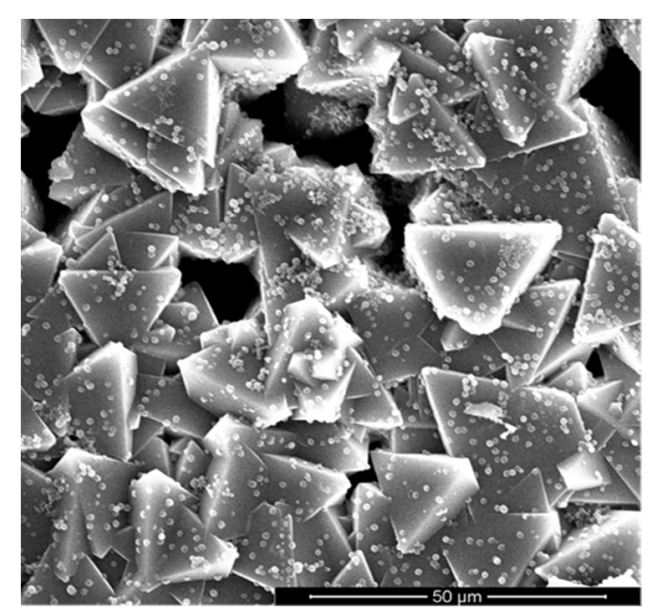

Figure 3. Top view SEM image of the NDC-Y-fcu-MOF thin film grown on the IDE substrate.

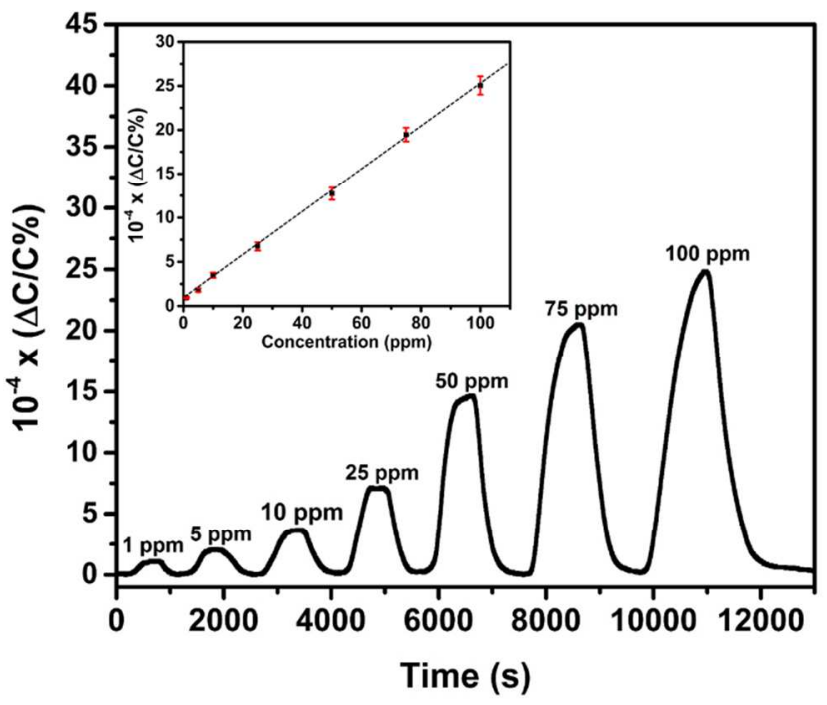

Figure 4. Detection of $\mathrm{NH}_{3}$ in different ranges of ppm concentrations: from (1-100 ppm). Insets: Linear response for the NDC-Yfcu-MOF in the corresponding range with error bars depicted in red.

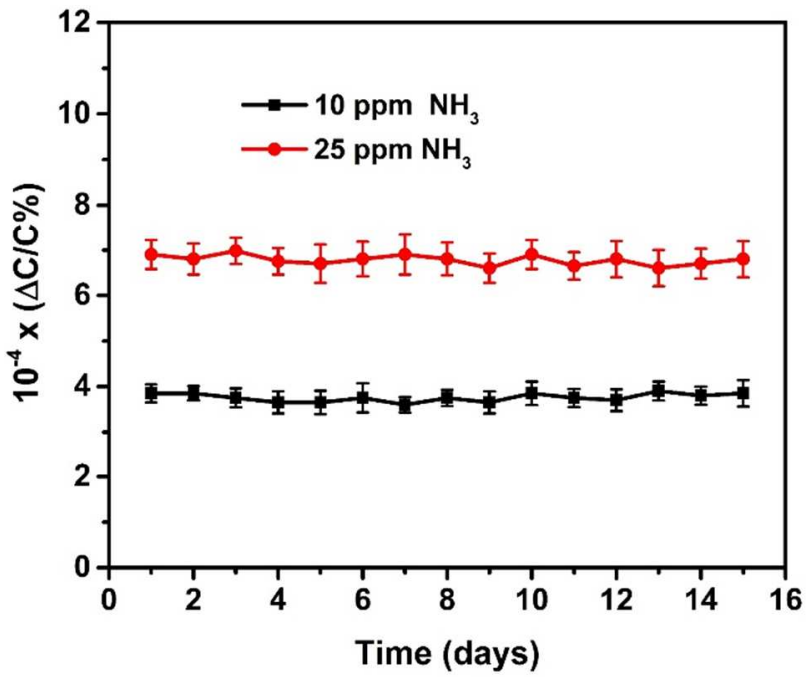

Figure 5. Stability performance for Y-NDC-fcu-MOF capacitive sensor upon exposure to 10 and 25 ppm of NH3 over a 15-days' time period.

The temperature (T) dependence on $\mathrm{NH}_{3}$ sensitivity for our NDC-Y-fcu-MOF capacitive sensor was investigated up to $80^{\circ} \mathrm{C}$, as shown in Figure 7, where a decrease in the sensitivity with the increase of the NDC-Y-fcu-MOF sensitive layer temperature was observed. The best sensitivity was obtained at room temperature, and shown to be lessened with the increase of temperature from $22{ }^{\circ} \mathrm{C}$ to $80{ }^{\circ} \mathrm{C}$. This drop in the sensitivity response of the sensor can be attributed to the reduced sorption/interaction capability of the active sites in the NDC-Y-fcu-MOF capacitive sensor at relatively elevated temperatures. The adsorption/interaction of the gas in the sensing film strongly decreases with the temperature, reducing thus the capacitance shift. This observed behavior is in good agreement with other reported gas sensors. ${ }^{51,54,55}$ 


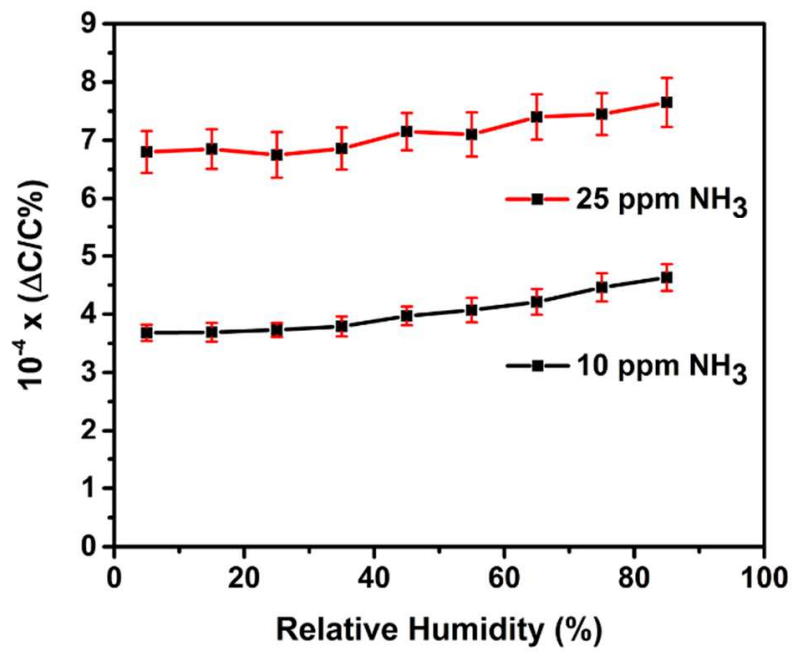

Figure 6. Stability of ammonia detection for Y-NDC-fcu-MOF upon exposure to 10 and $25 \mathrm{ppm}$ of $\mathrm{NH}_{3}$ at variable relative humidity $(\mathrm{RH}) \%$.

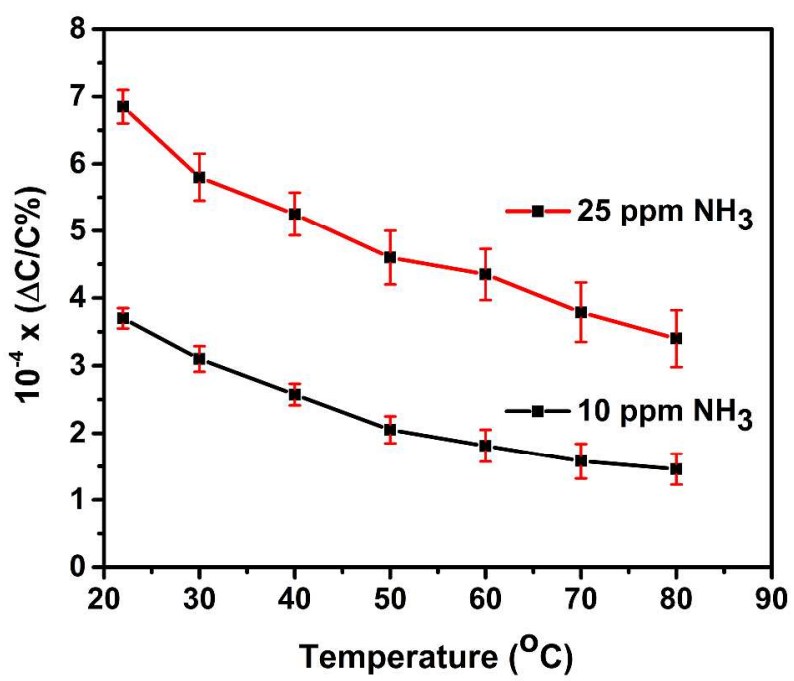

Figure 7. Stability of ammonia detection for Y-NDC-fcu-MOF upon exposure to 10 and $25 \mathrm{ppm}$ of $\mathrm{NH}_{3}$ at variable under different temperatures.

Appealingly, as depicted in Figure 8a, the NDC-Y-fcu-MOF proved to express excellent selectivity for $\mathrm{NH}_{3}$ in the presence of other tested gases/vapors, although a slight cross sensitivity with $\mathrm{NH}_{3}$ was observed in cases of $\mathrm{NO}_{2}, \mathrm{CH}_{4}$, and $\mathrm{C}_{7} \mathrm{H}_{8}$. As shown in Figure $8 \mathrm{a}$, the response signal of the NDC-Y-fcu-MOF to $\mathrm{NH}_{3}$ was nearly four-fold the corresponding signal for $\mathrm{NO}_{2}$, and noticeably negligible signals were observed for the other evaluated gases and vapors (e.g., $\mathrm{CH}_{4}, \mathrm{H}_{2}$, and $\mathrm{C}_{7} \mathrm{H}_{8}$ ). The observed enhanced selectivity of $\mathrm{NH}_{3}$ over $\mathrm{CH}_{4}$ and $\mathrm{H}_{2}$ can be attributed to the insignificant adsorption of the $\mathrm{CH}_{4}$ and $\mathrm{H}_{2}$ molecules by the MOF due to the lack of any interaction between the MOF surface and the nonpolar and weakly interacting molecules. On the hand the selectivity of $\mathrm{NH}_{3}$ over $\mathrm{C}_{7} \mathrm{H}_{8}$ and $\mathrm{C}_{3} \mathrm{H}_{8}$ is supported by the associated big size of these molecules, prohibiting their diffusion/passage through the triangular window of the evaluated fcuMOF. It is to be noted that the triangular opening is the sole entrance/access to the fcu-MOF pore system. In this case the poreaperture is not large enough to allow the gases/vapors to adsorb/diffuse into the pores without resistance. In addition, the $\mathrm{C}_{7} \mathrm{H}_{8}$ and $\mathrm{C}_{3} \mathrm{H}_{8}$ molecules do not have specific functionalities to promote interactions with the MOF.
On the other hand, the polar $\mathrm{NH}_{3}$ and $\mathrm{NO}_{2}$ molecules can easily be adsorbed by the MOF and interact with the MOF surface which encompasses polar hexanuclear moieities. The better selectivity of $\mathrm{NH}_{3}$ over $\mathrm{NO}_{2}$ can be attributed to the favorable interaction of $\mathrm{NH}_{3}$ lone pairs with the unsaturated metal sites exposed on the MOF pore system. In addition H-bonding between the adsorbed $\mathrm{NH}_{3}$ molecules can also help significant the adsorption of $\mathrm{NH}_{3}$ and hence the better observed sensitivity. These aforementioned features are not available in the case of the $\mathrm{NO}_{2}$ molecules and thus the less observed sensitivity for the evaluated fcu-MOF.

These results pinpoint the great sensing/selectivity of the NDCY-fcu-MOF for $\mathrm{NH}_{3}$ even in the presence of other gases/vapors with different physical and chemical properties. ${ }^{56,57}$

Comparatively and in order to bring to the fore the unique ammonia sensing performance of The NDC-Y-fcu-MOF, we assessed the sensing potential of distinct MOFs like HKUST-1, $\left.\mathrm{Cu}(\mathrm{bdc}) \cdot \mathrm{xH}_{2} \mathrm{O}\right)$ and $\mathrm{ZIF}-8$ for ammonia down to $1 \mathrm{ppm}$ under the same conditions. As clearly depicted in Figure $8 \mathrm{~b}$, the performance of the aforementioned other three MOFs was dramatically inferior to that of the NDC-Y-fcu-MOF. This observed poor performance is directly correlated to the degradation/low-stability of the evaluated MOF in the presence of ammonia, especially in the case of HKUST-1 and $\mathrm{Cu}(\mathrm{bdc}) \cdot \mathrm{xH}_{2} \mathrm{O}$. Specifically as evidenced and depicted in Figure 8b, the detection signal for the NDC-Yfcu-MOF is noticeably much higher (i.e., three times) than the signal for the other three tested MOFs, attesting to the distinctive high affinity of the NDC-Y-fcu-MOF for $\mathrm{NH}_{3}$. Markedly, the noted sensing from the realized tests corroborate the stability of the NDC-Y-fcu-MOF as an effective ammonia sensor with superior $\mathrm{NH}_{3}$ detection than the evaluated MOFs at room temperature, in accordance with the low $\mathrm{NH}_{3}$ stability of the associated bulk MOFs like HKUST-1 and $\mathrm{Cu}(\mathrm{bdc}) \cdot{ }_{\mathrm{X}} \mathrm{H}_{2} \mathrm{O}$ and the low $\mathrm{NH}_{3}$ affinity for ZIF-8. ${ }^{58}$ Comparing the performance of our NDC-Y-fcu-MOF capacitive sensor with the performance of other different sensors being applied in different fields, revealed that prominently, the NDC-Y-fcu-MOF offers the prospective to detect ammonia at room temperature and at a relatively low concentrations in contrast to the mixed metal oxide $\mathrm{WO}_{3}-\mathrm{SnO}_{2}$ hierarchical nanostructured material, necessitating a relatively higher temperature around $300^{\circ} \mathrm{C}$ for a detection limit of only about $400 \mathrm{ppm}$ of ammonia. ${ }^{21}$ In addition, our MOF based sensor revealed a comparable detection concentration of $1 \mathrm{ppm}$ as in the case of polyaniline nanoparticles, but at room temperature instead of $80^{\circ} \mathrm{C} .^{22}$ Moreover, our MOF based sensor performed better than optical detection method of ammonia, which is based on absorption of evanescent waves by organometallic reagent and was able to only detect down to $30 \mathrm{ppm} .{ }^{23}$ Notably, Our NDC-Y-fcu-MOF based sensor performed equally in the same concentration range reported (which is about $1 \mathrm{ppm}$ ) recently for some MOFs by Dinca et al. but at room temperature instead of at $100{ }^{\circ} \mathrm{C} .{ }^{41,42}$

Encouraged by the Y-NDC-fcu-MOF distinctive ammonia sensing capabilities, we assessed the performance of this unique MOF sensor for the ammonia detection using cross-over sensitivity testes like in a stimulated breathing system in the presence of a mixture of carbon dioxide $\left(\mathrm{CO}_{2}\right)$ and humidity.${ }^{14,49}$ We have tested the Y-NDC-fcu-MOF capacitive sensor using two concentrations of $\mathrm{NH}_{3} 1$ and $10 \mathrm{ppm}$. Certainly the results as depicted in Figure 9, revealed the excellent performance of the NDC-Y-fcuMOF sensing ammonia in the presence of the mixture of carbon dioxide and/or humidity (water vapor), with no major alteration in the detection signal being observed for both evaluated ammonia of $1 \mathrm{ppm}$ and $10 \mathrm{ppm}$. Tests on $\mathrm{CO}_{2}$ and water vapor separately, with same previous amounts, results not presented here, showed also very good stability. Remarkably, the stability of the NDC-Yfcu-MOF sensor for $\mathrm{NH}_{3}$ detection at room temperature was validated by performing reproducibility cycles (the inset of Figure 9), 
evidently confirming that the detections levels were steady/stable and uniform over the range of various cycles.
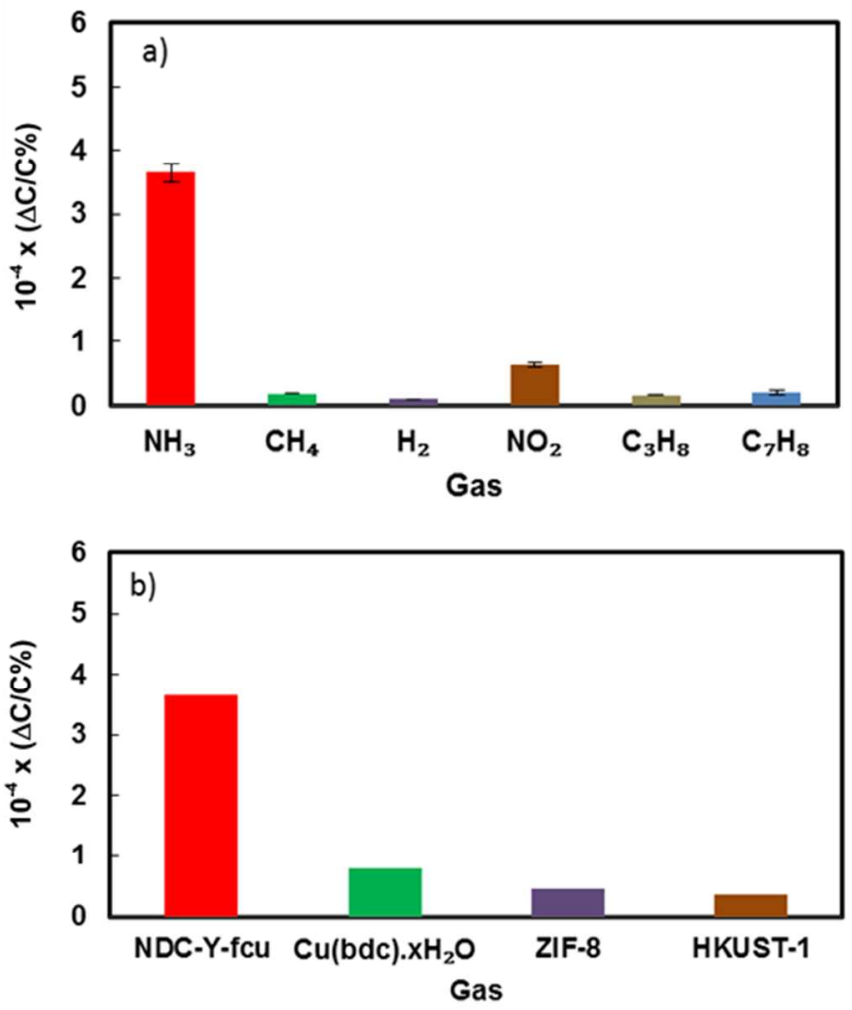

Figure 8. (a) Selectivity of the NDC-Y-fcu-MOF sensor to $\mathrm{NH}_{3}$ with respect to, $\mathrm{CH}_{4}, \mathrm{H}_{2}, \mathrm{NO}_{2}, \mathrm{C}_{3} \mathrm{H}_{8}$ and $\mathrm{C}_{7} \mathrm{H}_{8}$ at $1 \mathrm{ppm}$. (b) Selectivity of the NDC-Y-fcu-MOF sensor to $\mathrm{NH}_{3}$ with respect to $\mathrm{Cu}(\mathrm{bdc}) \cdot \mathrm{xH}_{2} \mathrm{O}, \mathrm{ZIF}-8$ and HKUST-1.

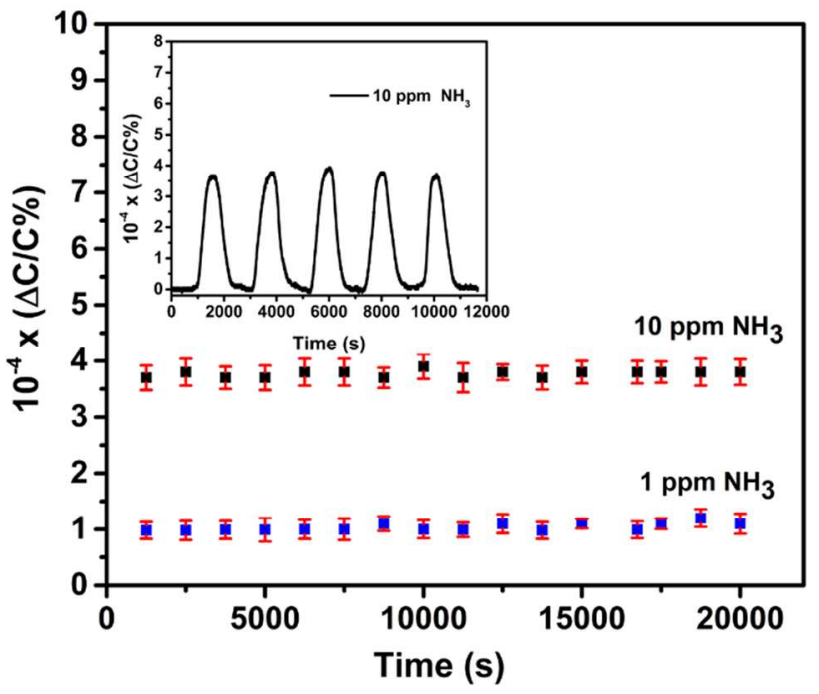

Figure 9. Stability of ammonia detection for Y-NDC-fcu-MOF at $1 \mathrm{ppm}$ and $10 \mathrm{ppm}$ in the presence of $5.3 \mathrm{CO}_{2} \%+6.2 \% \mathrm{H}_{2} \mathrm{O}$. Inset: reproducibility test of the sensor at $10 \mathrm{ppm}$ of Ammonia
The performance of the NDC-Y-fcu-MOF as a sensing layer on a capacitive IDE sensor, for $\mathrm{NH}_{3}$ detection at room temperature was successfully demonstrated. Principally, the NDC-Y-fcu-MOF offers a distinctive $\mathrm{NH}_{3}$ detection down to concentrations down to around $1 \mathrm{ppm}$ with a limit of detection of about $100 \mathrm{ppb}$. The stability of the NDC-Y-fcu-MOF sensor was affirmed and supported using different methods, corroborating its excellent chemical stability as compared to various other MOFs, e.g. ZIF-8 and $\mathrm{Cu}(\mathrm{bdc}) \cdot \mathrm{xH}_{2} \mathrm{O}$. Markedly, the NDC-Y-fcu-MOF distinct sensing prospective for ammonia was sustained with precision, even at relatively low concentration, in the presence of various gases and/or vapors such as water vapor, carbon dioxide nitrogen oxide, methane, hydrogen, and toluene. The presented NDC-Y-fcu-MOF exhibited a comparable stability with time and under variable humidity conditions compared to other reported sensors. The NDC-Y-fcu-MOF based sensor exhibited excellent performance for sensing ammonia for simulated breathing system, with no major alteration in the detection signal being observed. Prominently, the revealed distinctive and remarkable sensing selectivity of the NDC-Y-fcu-MOF for $\mathrm{NH}_{3}$ paves the way for the deployment of MOFs as practical sensors for various prominent applications.

\section{AUTHOR INFORMATION}

\section{Corresponding Author}

Email:

mohamed.eddaoudi@kaust.edu.sa, khaled.salama@kaust.edu.sa

\section{Author Contributions}

\#these authors contributed equally.

\section{Notes}

The authors declare no competing financial interests.

\section{ACKNOWLEDGMENT}

The authors would like to thank Dr Y. Belmabkhout, Dr. H. Omran, and Mr C. Sapsanis for their technical support and helpful advice. This work was partially sponsored by the Advanced Membranes and Porous Materials (AMPM) FCC project, FCC/1/1972-05-01.

\section{REFERENCES}

(1) Warneck, P. Chemistry of the Natural Atmosphere, Academic Press Inc., 1998.

(2) Bouwman, A. F.; Lee, D. S.; Asman, W. A. H.; Dentener, F. J.; Van der Hoek, K. W.; Olivier, J. G. J. A global high-resolution emission inventory for ammonia Global Biogeochemical Cycles, 1997, 11, 561-587.

(3) Behera, S. N. ; Sharma, M. ; Aneja, V. P. and Balasubramanian, R. Ammonia in the atmosphere: a review on emission sources, atmospheric chemistry and deposition on terrestrial bodies Environ. Sci. Pollut. R., 2013, 20, 8092-8131.

(4) Riddick, S.; Ward, D.; Hess, P.; Mahowald, N.; Massad, R.; Holland, E. Estimate of changes in agricultural terrestrial nitrogen pathways and ammonia emissions from 1850 to present in the Community Earth System Model Biogeosciences, 2016, 13, 3397-3426.

(5) Sapek, A. Ammonia emissions from non-agricultural sources Pol. J. of Environ. Stud., 2013, 22, 63-70.

(6) Krupa, S. V. Effects of atmospheric ammonia $\left(\mathrm{NH}_{3}\right)$ on terrestrial vegetation: a review Environ. Pollut. 2003, 124, 179-221.

(7) Istas, J. R.; de Borger, R.; de Temmerman, L.; Guns, Meeus-Verdinne, K.; Ronse, A.; Scokart, P.; Termonia, M. European Communities Report No. EUR 11857 EN, 1988.

(8) Timmer, B.; Olthuis, W.; van den Berg, A. Ammonia sensors and their applications - a review Sens. Actuators B., 2005, 107, 666-677.

(9) Erisman, J. W.; Otjes, R.; Hensen, A.; Jongejan, P.; Bulk, P.v.d.; Khlystov, A.; Mols, H.; Slanina, S. Instrument development and applica-

\section{CONCLUSIONS}


tion in studies and monitoring of ambient ammonia Atmos. Environ., 2001, 35, 1913-1922.

(10) Sberveglieri, G. Recent developments in semiconducting thin-film gas sensors Sens. Actuators B-Chem., 1995, 23, 103-109.

(11) Clifford, P. K.; Tuma, D.T. Characteristics of semiconductor gas sensors I. Steady state gas response. Sens. Actuators. 1983, 3, 233-254.

(12) Srivastava, R.K.; Lal, P.; Dwivedi, R.; Srivastatva, S.K. Sensing mechanism in tin oxide based thick film gas sensors Sens. Actuators BChem., 1994, 21, 213-218.

(13) Huebner H. P.; Drost, S. Tin oxide gas sensors: An analytical comparison of gas-sensitive and non-gas-sensitive thin films Sens. Actuators B-Chem., 1991, 4, 463-466.

(14) Imawan, C.; Solzbacher, F.; Steffes H.; Obermeier, E. Gas-sensing characteristics of modified- $\mathrm{MoO}_{3}$ thin films using Ti-overlayers for $\mathrm{NH}_{3}$ gas sensors Sens. Actuators B-Chem., 2000, 64, 193-197.

(15) Winquist, F.; Spetz, A.; Lundstrom, I. Determination of ammonia in air and aqueous samples with a gas-sensitive semiconductor capacitor Anal. Chim. Acta, 1984, 164, 127-138.

(16) Lundstrom, I.; Spetz, A.; Winquist, F.; Ackelid, U.; Sundgren, H. Catalytic metals and field-effect devices - a useful combination Sens. Actuators B-Chem., 1990, 1, 15-20.

(17) Kukla, A. L.; Shirshov, Y. M.; Piletsky, S. A. Ammonia sensors based on sensitive polyaniline films Sens. Actuators. B-Chem., 1996, 37, $135-140$.

(18) Chabukswar, V. V.; Pethkar, S.; Athawale, A. A. Acrylic acid doped polyaniline as an ammonia sensor Sens. Actuat. B-Chem., 2001, 77, $657-$ 663.

(19) Vogel, A. I. Vogel's Qualitative Inorganic Analysis, 6th ed., Longman Scientific \& Technical, 1987.

(20) Ghauch, A.; Rima, J.; Charef, A.; Suptil, J.; Fachinger, C.; Martin-

Bouyer, M. Quantitative measurements of ammonium, hydrogenophosphate and $\mathrm{Cu}(\mathrm{II})$ by diffuse reflectance spectrometry Talanta, 1999, 48, $385-392$.

(21) Nayak, A. K.; Ghosh, R.; Santra, S.; Guha, P. K.; Pradhan, D. Hierarchical nanostructured $\mathrm{WO}_{3}-\mathrm{SnO}_{2}$ for selective sensing of volatile organic compounds Nanoscale, 2015, 7, 12460-12473.

(22) Crowley, K.; Morrin, A.; Hernandez, A.; O'Malley, E.; Whitten, P. G.; Wallace, G. G.; Smyth, M. R.; Killard, A. J. Fabrication of an ammonia gas sensor using inkjet-printed polyaniline nanoparticles Talanta, 2008, 77, 710-717.

(23) Aubrecht, J.; Kalvoda, L. Development of ammonia gas sensor using optimized organometallic reagent $J$. Sens., 2016, 2, 1-8.

(24) Yavari, F.; Chen, Z. P.; Thomas, A. V.; Ren, W. C.; Cheng, H. M.; Koratkar, N. High sensitivity gas detection using a macroscopic threedimensional graphene foam network Sci. Rep., 2011, 1, 5-9.

(25) Duy, L. T.; Trung, T. Q.; Dang, V. Q.; Hwang, B. -U.; Siddiqui, S.; Son, I. Y.; Yoon, S. K.; Chung, D. J.; Lee, N. E. Flexible transparent reduced graphene oxide sensor coupled with organic dye molecules for rapid dual-mode ammonia gas detection $A d v$. Func. Mat., 2016, 26, 43294338.

(26) Omran, H.; Salama, K.N. IEEE International Conference on Smart Instrumentation, Measurement and Application, Design and fabrication of capacitive interdigitated electrodes for smart gas sensors 201510.1109 / ICSIMA.2015.7559021.

(27) Tsoutia, V.; Boutopoulosb, C.; Zergiotib, I.; Chatzandroulis, S. Capacitive microsystems for biological sensing Biosens. Bioelectron., 2011, 27, $1-11$.

(28) Llobet, E. Gas sensors using carbon nanomaterials: A review Sens. Actuators B., 2013, 179, 32-45.

(29) Pavinattoa, F. J.; Carlos, W.; Paschoalc, A.; Ariasa, A.-C. Printed and flexible biosensor for antioxidants using interdigitated ink-jetted electrodes and gravure-deposited active layer Biosens. Bioelectron., 2015, 67, $553-559$.

(30) Choi, S. J.; Choi, C.; Kim, S. J.; Cho, H. J.; Hakim, M.; Jeon, S.; Kim, I-D. Highly efficient electronic sensitization of non-oxidized graphene flakes on controlled pore-loaded $\mathrm{WO}_{3}$ nanofibers for selective detection of $\mathrm{H}_{2} \mathrm{~S}$ molecules Sci. Rep., 2015, 5, 8067-8075.

(31) Mashraei, Y.; Sivashankar, S.; Buttner, U.; Salama, K. N. Integration of fractal biosensor in a digital microfluidic platform IEEE Sens J., 2016, $16,8775-8783$.

(32) Sivashankar, S.; Sapsanis, C.; Buttner, U.; Salama, K. N. Flexible low-cost cardiovascular risk marker biosensor for point-of-care applications Electron. Lett., 2015, 51, 1746-1747.

(33) Kuppler, R. J.; Timmons, D. J.; Fang, Q. R.; Li, J. R.; Makal, T. A.; Young, M. D.; Yuan, D. Q.; Zhao, D.; Zhuang, W. J.; Zhou, H. C. Poten- tial applications of metal-organic frameworks Coord. Chem. Rev., 2009, $253,3042-3066$

(34) Kreno, L. E.; Leong, K.; Farha, O. K.; Allendorf, M.; Duyne, R. P. V.; Hupp, J. T. Metal-organic framework materials as chemical sensors Chem. Rev., 2012, 112, 1105-1125.

(35) Xue, D. X.; Belmabkhout, Y.; Shekhah, O.; Jiang, H.; Adil, K.; Cairns, A. J.; Eddaoudi, M. Tunable Rare Earth fcu-MOF platform: Access to adsorption kinetics driven gas/vapor separations via pore size contraction J. Am. Chem. Soc., 2015, 137, 5034-5040.

(36) Xue, D, X.; Cairns, A. J.; Belmabkhout, Y.; Wojtas, L.; Liu, Y.; Alkordi, M. H.; Eddaoudi, M. Tunable Rare-Earth fcu-MOFs: A platform for systematic enhancement of $\mathrm{CO}_{2}$ adsorption energetics and uptake $J$. Am. Chem. Soc., 2013, 135, 7660-7667.

(37) Assen, A. H.; Belmabkhout, Y.; Adil, K.; Bhatt, P. M.; Xue, D. X.; Jiang, H.; Eddaoudi, M. Ultra-tuning of the Rare-Earth fcu-MOF aperture size for selective molecular exclusion of branched paraffins Angew. Chem. Int. Ed., 2015, 54, 14353-14358.

(38) Yassine, O.; Shekhah, O.; Assen, A. H.; Belmabkhout, Y.; Salama, K. N.; Eddaoudi, M. $\mathrm{H}_{2} \mathrm{~S}$ Sensors: Fumarate-based fcu-MOF thin film grown on a capacitive interdigitated electrode Angew. Chem. Int. Ed., 2016, 55, $15879-15883$.

(39) Sapsanis, C.; Omran, H.; Chernikova, V.; Shekhah, O.; Belmabkhout, Y.; Buttner, U.; Eddaoudi, M.; Salama, K. N. Insights on capacitive interdigitated electrodes coated with MOF thin films: Humidity and VOCs sensing as a case study Sensors, 2015, 15,18153-18166.

(40) Omran, H.; Arsalan, M.; Salama, K. N. A robust parasitic-insensitive successive approximation capacitance-to-digital converter IEEE Custom Integrated Circuits Conference (CICC), 2014.

(41) Shustova, N. B.; Cozzolino, A. F.; Reineke, S.; Baldo, M.; Dinca, M. Selective turn-on ammonia sensing enabled by high-temperature fluorescence in metal-organic frameworks with open metal sites $J$. Am. Chem. Soc., 2013, 135, 13326-13329.

(42) Campbell, M. G.; Sheberla, D.; Liu, S. F.; Swager, T. M.; Dinca, M. $\mathrm{Cu}_{3}$ (hexaiminotriphenylene) $)_{2}$ : an electrically conductive 2D metal-organic framework for chemiresistive sensing Angew. Chem. Int. Ed., 2015, 54, $4349-4352$.

(43) Hester, J. G. D.; Tentzeris, M. M.; Fang, Y. Proceedings of the 45th European Microwave Conference, 2015, 978-2-87487-039-2.

(44) Shekhah, O.; Busse, C.; Bashir, A.; Turcu, F.; Yin, X.; Cyganik, P.; Birkner, A.; Schuhmann, W.; Wöll, C. Electrochemically deposited Pd islands on an organic surface: the presence of Coulomb blockade in STM $I(V)$ curves at room temperature Phys. Chem. Chem. Phys., 2006, 29,33753378.

(45) Shekhah, O.; Roques, N.; Mugnaini, V.; Munuera, C.; Ocal, C.; Veciana, J.; Wöll, C. Grafting of monocarboxylic substituted polychlorotriphenylmethyl radicals onto a $\mathrm{COOH}-$ functionalized self-assembled monolayer through Copper (II) metal ions Langmuir, 2008, 24, 6640-6648.

(46) Streit, H.; Adlung, M.; Shekhah, O.; Stammer, X.; Arslan, H. K.; Zybaylo, O.; Ladnorg, T.; Gliemann, H.; Franzreb, M.; Wöll, C.; Wickleder, C. Surface-anchored MOF-based photonic antennae Chem. Phys. Chem., 2012, 13, 2699-2702.

(47) Alammar, T.; Shekhah, O.; Wohlgemuth, J.; Mudring, A. V. Ultrasound-assisted synthesis of mesoporous $\beta-\mathrm{Ni}(\mathrm{OH})_{2}$ and $\mathrm{NiO}$ nano-sheets using ionic liquids J. Mater. Chem., 2010, 22, 18252-18260.

(48) Shekhah, O.; Fu, L.; Sougrat, R.; Belmabkhout, Y.; Cairns, A. J.; Giannelis, E. P.; Eddaoudi, M. Successful implementation of the stepwise layer-by-layer growth of MOF thin films on confined surfaces: mesoporous silica foam as a first case study Chem. Commun., 2012, 48, 11434-11436.

(49) Shekhah, O.; Eddaoudi, M. The liquid phase epitaxy method for the construction of oriented ZIF-8 thin films with controlled growth on functionalized surfaces Chem. Commun., 2013, 49, 10079-10081.

(50) Hu, M.; Kang, W.; Li, Z.; Jie, S.; Zhao, Y.; Li, L.; Cheng, B. Zinc(II)porphyrin-poly(lactic acid) nanoporous fiber membrane for ammonia gas detection J. Porous Mater., 2016, 23, 911-917.

(51) Li, J.; Lu, Y.; Ye, Q.; Cinke, M.; Han, J.; Meyyappan, M. Carbon Nanotube Sensors for Gas and Organic Vapor Detection Nano Lett. 2003, 3, 929-933.

(52) Yi, F.Y.; Chen, D.; Wu, M. K.; Han, L.; Jiang, H. L. Chemical sensors based on Metal-Organic Frameworks ChemPlusChem, 2016, 81,675690 .

(53) Bannov, A. G.; Prášek, J.; Jašek, O.; Zajǐ̌cková, L. Investigation of pristine graphite oxide as room-temperature chemiresistive ammonia gas sensing material Sensors, 2017, 17, 320; doi:10.3390/s17020320. 
(54) Grate, J. W.; Abraham, M. H. Solubility interactions and the design of chemically selective sorbent coatings for chemical sensors and arrays Sensors and Actuators, B, 1991. 3, 85-111.

(55) Carquigny, S.; Sanchez, J.-B.; Berger, F.; Lakard, B.; Lallemand, F. Ammonia gas sensor based on electrosynthesized polypyrrole films Talanta, 2009, 78, 199-206.

(56) Samotaev, N. N.; Podlepetsky, I.; Vasiliev, A.; Pisliakov, V.; Sokolov, V. Metal-oxide gas sensor high-selective to ammonia Automation and Remote Control,_2013, 74, 308-312.

(57) Dai, L.; Meng, W.; Meng, W.; Zhou, H.; Yang, G.; Li, Y.; Wang, L. An impedancemetric $\mathrm{NH}_{3}$ sensor based on $\mathrm{La}_{10} \mathrm{Si}_{5} \mathrm{MgO}_{26}$ electrolyte and nano-structured $\mathrm{CoWO}_{4}$ sensing electrode J. Electrochem. Soc., 2016, 163, B1-B7.

(58) Kajiwara, T.; Higuchi, M.; Watanbe, D.; Higashimura, H.; Yamada, T.; Kitagawa, H. A systematic study on the stability of porous coordination polymers against ammonia Chem. Eur. J., 2014, 20, 15611-15617. 
"for TOC only"

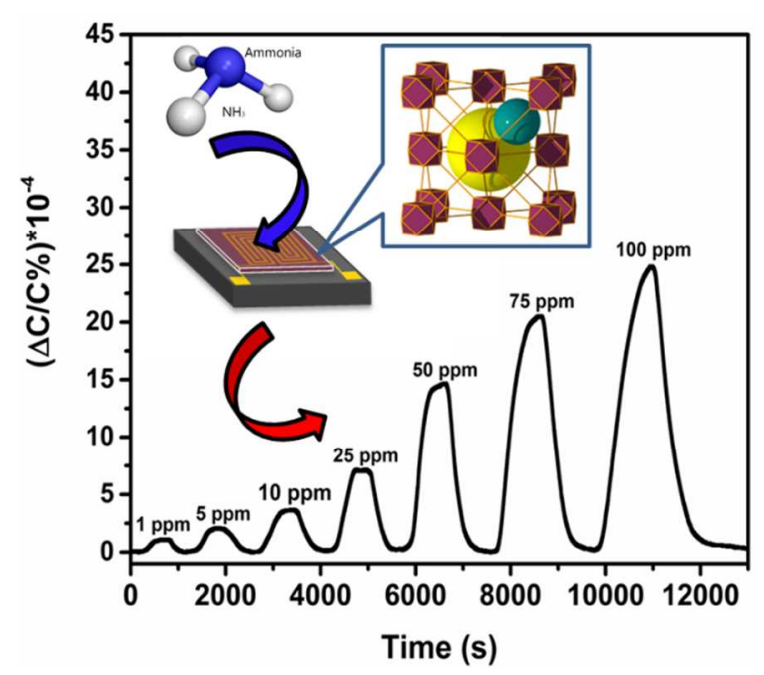

This work reports on the fabrication of an advanced sensor using thin films of rare-earth based metalorganic frameworks (MOFs) for the detection of toxic gas, namely ammonia $\left(\mathrm{NH}_{3}\right)$ at room temperature. The NDC-Y-fcu-MOF sensor exhibits the highly desirable detection selectivity towards $\mathrm{NH}_{3}$ vs. $\mathrm{CH}_{4}, \mathrm{NO}_{2}, \mathrm{H}_{2}$ and $\mathrm{C}_{7} \mathrm{H}_{8}$ as well as an outstanding $\mathrm{NH}_{3}$ sensing stability as compared to other reported MOFs. 